\title{
Rare case of Cushing's disease due to double ACTH-producing adenomas, one located in the pituitary gland and one into the stalk
}

\author{
Marco Mendola, ${ }^{1}$ Alessia Dolci, ${ }^{1}$ Lanfranco Piscopello, ${ }^{2}$ Giustino Tomei, ${ }^{3}$ Dario Bauer, ${ }^{4}$ \\ Sabrina Corbetta, ${ }^{1}$ Bruno Ambrosi ${ }^{1}$ \\ ${ }^{1}$ Endocrinology and Diabetology Unit, Department of Biomedical Sciences for Health, University of Milan, IRCCS \\ Policlinico San Donato, San Donato M.se, Milano; ${ }^{2}$ Endocrinology Unit, Ospedale Maggiore Trieste; ${ }^{3}$ Neurosurgical \\ Unit, Department of Biotechnology, University of Insubria, Varese; ${ }^{4}$ Pathology Unit, Department of Health Sciences, \\ University of Milan, San Paolo Hospital, Milano; Italy
}

\begin{abstract}
We describe a patient affected by Cushing's disease due to the presence of double pituitary adenomas, one located within the anterior pituitary and the other in the infundibulum associated with a remnant of Rakthe's pouch. Cure was achieved only after the infundibulum lesion was surgically removed. CASE REPORT: A 38-year-old female presented with unexplained weight gain, hirsutism, amenorrhea, asthenia, recurrent cutaneous micotic infections and alopecia. Hormonal studies indicated Cushing's disease and MRI showed an enlarged pituitary gland with a marked and homogeneous enhancement after injection of gadolinium and an enlarged infundibulum with a maximum diameter of $8 \mathrm{~mm}$. As a venous sampling of the inferior petrosal sinus after $10 \mu \mathrm{g}$ iv desmopressin stimulation revealed a central to peripheral ACTH ratio consistent with a pituitary $\mathrm{ACTH}$-secreting tumor, transphenoidal explorative surgery was performed and a 4-mm pituitary adenoma immunopositive for ACTH was disclosed and removed. Since postoperative hormonal evaluation showed persistent hypercortisolism, confirmed by dynamic tests, the patient again underwent surgery by transcranial access and the infundibulum mass was removed. Histology and immunochemistry were consistent with an ACTH-secreting adenoma. A few months after the second operation, cushingoid features were significantly reverted and symptoms improved. CONCLUSION: Although Cushing's patients bearing multiple adenomas have already been documented, the presence of two adenomas both immunohistochemically positive for ACTH is a very rare cause of Cushing's disease and this is the first report of a case of double ACTH-producing adenomas, one located in the pituitary gland and one attached to the stalk.
\end{abstract}

Key words: Cushing's disease, Double ACTH-producing adenomas, Magnetic resonance imaging, Pituitary stalk 


\section{INTRODUCTION}

Multiple pituitary adenomas are defined as simultaneous, morphologically or immunocytologically, distinct tumors ${ }^{1}$ and are classified as contiguous and clearly separated double tumors. These tumors are rarely detected among surgical specimens (0.004$0.01 \%$ ), the most frequent clinical manifestation in the reported patients being acromegaly. ${ }^{2}$ The diagnosis of double pituitary adenomas is, in most cases, based on histopathologic examination, since preoperative evaluation and MRI are able to identify multiple adenomas in only a few cases. These adenomas can belong to the same or to different hormone groups, making different combinations possible: ACTH-PRL secreting tumors appear to be the most common, followed by $\mathrm{GH}$-non functioning adenomas and $\mathrm{GH}$ PRL adenomas. ${ }^{2}$ Herein we describe, to the best of our knowledge, the first case in the recent literature of a middle-aged woman with Cushing's disease due to double adenomas, one located within the anterior pituitary and the other in the infundibulum.

\section{CASE PRESENTATION}

A 38-year-old woman was referred to our Endocrinology Unit as she had shown over the last three years progressive unexplained weight gain $(>25 \mathrm{Kg}$ bw) and hirsutism. Secondary amenorrhea had been present for six months. A polycystic ovary syndrome had been previously diagnosed and she was enrolled in a weight reduction program, without, however, any improvement. Moreover, one year before she developed typical hypercortisolemic clinical features including weight gain, depression, alopecia, profound asthenia, recurrent cutaneous micotic infections. She denied current use of alcohol. She did not suffer from other multiple endocrine neoplasia (MEN1) or Carney complex clinical manifestations.

On physical examination, vital signs were normal, weight was $90 \mathrm{~kg}$ and height was $169 \mathrm{~cm}$ (BMI 31.5 $\mathrm{kg} / \mathrm{m}^{2}$ ). Other clinical signs, e.g. moon face with mild plethora, truncal obesity, alopecia, thin skin with many bruises and abdominal striae, suggested the presence of Cushing's syndrome.

Her morning plasma ACTH and serum cortisol levels were $40-70 \mathrm{pg} / \mathrm{ml}$ and $497-821 \mathrm{nmol} / \mathrm{L}$, respec- tively. Both overnight low dose $(1 \mathrm{mg})$ dexamethasone (DXM) and low dose (2 mg for 2 days) DXM test showed an inadequate suppression of her serum cortisol (390 nmol/L/dl and $355 \mathrm{nmol} / \mathrm{L}$, respectively; normal suppression $<50 \mathrm{nmol} / \mathrm{L}$ ). Hypercortisolism was further confirmed by elevated urinary free cortisol (407-960 nmol/24h; normal range $35-275 \mathrm{nmol} / 24 \mathrm{~h}$ ). High dose dexamethasone suppression test $(8+8$ $\mathrm{mg}$ for 2 days) revealed suppression of both serum cortisol (from 868 to $108 \mathrm{nmol} / \mathrm{L}$ ) and UFC (urinal free cortisol) levels (from 960 to $59 \mathrm{nmol} / 24 \mathrm{~h}$ ). A corticotropin releasing hormone test (CRH; $1 \mathrm{mcg}$ / $\mathrm{kg} \mathrm{bw}$ ) revealed greatly increased levels of both cortisol and ACTH (by $+76 \%$ and $+92 \%$, respectively, over baseline).

A positive response to ACTH (from 43 to 112 $\mathrm{pg} / \mathrm{ml},+160 \%$ ) and cortisol levels (from 507 to 757 $\mathrm{nmol} / \mathrm{L},+49 \%$ ) was found also after desmopressin stimulation (DDAVP; $10 \mathrm{mcg}$ i.v.).

Magnetic resonance imaging (MRI) of the hypothalamic-pituitary region revealed an enlarged pituitary gland with a marked and homogeneous enhancement after gadolinium i.v. Indeed, the infundibulum was enlarged with a maximum diameter of $8 \mathrm{~mm}$ and impinged on the optic chiasm (Figure 1). Visual field evaluation revealed a minimal peripheral loss. As MRI did not identify a typical intrapituitary tumor, the patient underwent bilateral inferior petrosal sinus sampling during desmopressin stimulation. The central to peripheral ratio of ACTH was 6.17 on the

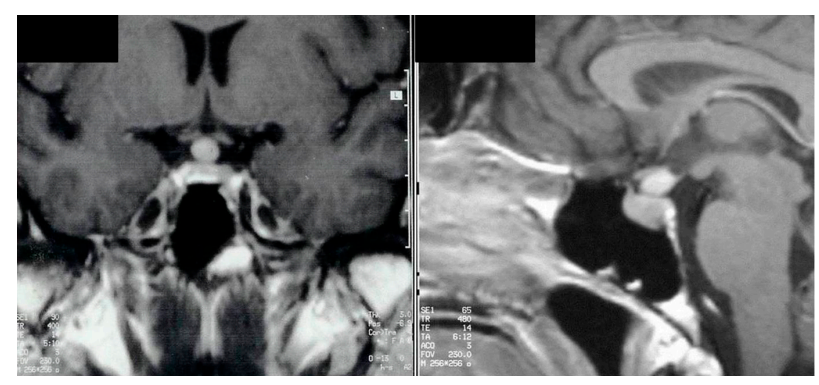

Figure 1. On the left, coronal T1-weighted, contrast-enhanced MR imaging showing a rounded mass located at the level of the pituitary stalk, over the pituitary gland, that appears intact; on the right, sagittal T1-weighted, contrast-enhanced MR imaging confirming the presence of a round mass located at the level of the pituitary stalk, over the pituitary gland, which in this plane seems enlarged in volume. 
left side after desmopressin, suggesting a possible corticotropinoma on that side. No pituitary hormones deficiencies were present.

The patient underwent transsphenoidal surgery to search for an intrapituitary microadenoma. After careful exploration of the pituitary gland, a grey soft tumor was identified and completely removed. The surgical specimen consisted of $4 \mathrm{~mm}$ of tissue and microscopically showed fragments of pituitary adenoma. The adenoma cells were immunoreactive for ACTH (Figure 2A) and immunonegative for $\mathrm{LH}$, FSH, GH, PRL and chromogranin A.

Despite these findings, postoperative hormonal evaluation showed persistent hypercortisolism.

Thereafter, based on the preoperative MRI findings, we strongly suspected that the lesion observed in the enlarged infundibulum might be a further source of ACTH secretion. Thus, one month later the patient underwent a second operation in order to remove the infundibulum lesion by transcranic access. Histological examination revealed remnants of Rakthe's pouch associated with a pituitary microadenoma. Immunohistochemical staining again demonstrated a positive reaction for ACTH (Figure 2B) and negative ones for LH, FSH, PRL, GH and chromogranin A.

Soon after the operation, ACTH and cortisol levels decreased to $4-7 \mathrm{pg} / \mathrm{ml}$ and $11-57 \mathrm{nmol} / \mathrm{L}$, respectively, (Table 1). In the subsequent days, weakness, fatigue, hypotension and skin pallor, indicative of a hypoadrenal condition, progressively ensued. As signs of central hypopituitarism $(\mathrm{LH}<0.1 \mathrm{mIU} / \mathrm{ml}$, FSH $<0.1 \mathrm{mIU} / \mathrm{ml}$, TSH $0.01 \mu \mathrm{UI} / \mathrm{ml}$ ) appeared, hormonal replacement therapy was initiated, including cortisone acetate, l-thyroxine, desmopressin and estro-progestinic therapy.

\section{DISCUSSION}

We herein describe a patient with Cushing's disease due to the presence of two ACTH-secreting adenomas, one located within the anterior pituitary and the other in the infundibulum.

Very recently, an incidence of $2.6 \%$ of double pituitary adenomas in an unselected surgical series of 117 patients undergoing surgery for pituitary adenoma was reported. ${ }^{3}$ Previously, Kontogeorgos et al ${ }^{4}$
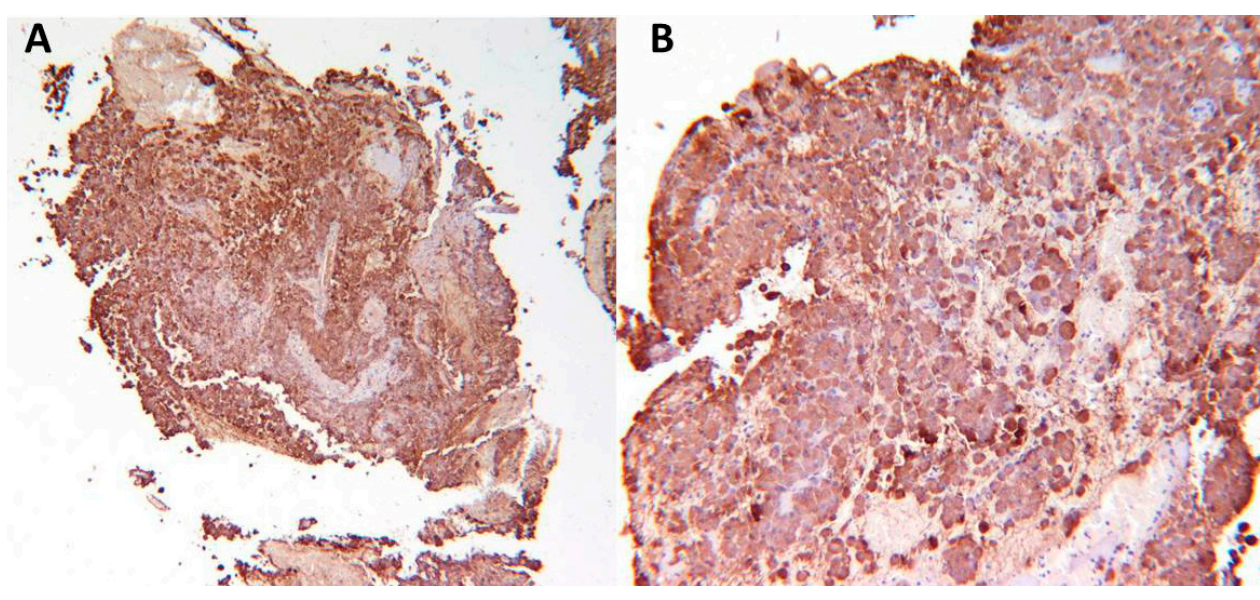

Figure 2. A: Intrapituitary microadenoma: adenomatous cells with positive immunochemistry for ACTH (4×); B: Adenoma attached to the stalk: adenomatous cells with positive immunostaining for ACTH (10×).

Table 1. Serum cortisol and plasma ACTH levels before and after surgery

\begin{tabular}{llcccc}
\hline & & Before surgery & $\begin{array}{c}\text { After 1 }^{\text {st }} \text { surgical } \\
\text { operation }\end{array}$ & $\begin{array}{c}\text { After 2 } \\
\text { operation }\end{array}$ & Normal range \\
\hline \multirow{2}{*}{ Cortisol $(\mathrm{nmol} / \mathrm{l})$} & Basal & 503 & 420 & $<28$ & $140-700$ \\
& After DXM 1mg & 390 & - & $<28$ & $<50$ \\
$\mathrm{ACTH}(\mathrm{pg} / \mathrm{ml})$ & & 43 & 71 & 7.7 & $10-90$ \\
\hline
\end{tabular}


showed that $8.9 \%$ of adenomas found at autopsy were multiple and, overall, $0.9 \%$ of autopsies detected the presence of multiple pituitary adenomas. It has also been emphasized that these tumors may occasionally co-exist with other brain tumors ${ }^{1,5-9}$ and that they may be distinguished as contiguous and clearly separated double tumors.

In autopsy specimens of double adenomas, prolactin was the most common immunoreactive secreted hormone, ${ }^{4,10}$ while most of the double adenomas in surgical series were a combination of $\mathrm{GH}$-secreting and clinically non-functioning adenomas: ${ }^{1,7,11-13}$ in fact, acromegaly has been shown to be the most frequent clinical feature in surgical series of double pituitary adenomas. ${ }^{1,5,7}$ Patients with somatotropinoma plus gonadotroph adenoma and cases of prolactinoma associated with $\mathrm{GH}$-secreting adenoma have also been described. ${ }^{1,714}$ It is of note that multiple (as many as three) intrapituitary non-functioning adenomas have additionally been documented. ${ }^{15}$

By contrast, immunostaining for adrenocorticotropic hormone in surgically resected double adenomas have rarely been described and these corticotroph adenomas were either "silent" or clinically active. In any case, isolated cases of Cushing's disease patients with double ${ }^{4,6,7,16}$ and also triple ${ }^{17}$ adenomas have been reported.

As far as double adenomas are concerned, ACTHsecreting pituitary adenomas have been described together with silent prolactinomas in two cases, ${ }^{16,17}$ with active PRL-secreting adenomas in four cases, $1,2,6,16$ with a somatotropinoma associated with acromegaly in one patient ${ }^{6}$ and with a silent $\mathrm{FSH}$-secreting adenoma in another one. ${ }^{18}$ Only one case of double pituitary adenomas ACTH-secreting has been reported recently in the literature. ${ }^{19}$

Owing to the rare occurrence of double pituitary adenomas, their pathogenesis is not known, but several possible mechanisms may be considered. One is a multicentric origin in the same pituitary, i.e. real double adenoma. The cause of multicentric adenomas might include incidental occurrence, promotion of the second adenoma growth through autocrine/paracrine pathways and a common origin. For example, studies on transgenic mice reported that several pituitary-driven growth factors can induce pituitary hyperplasia. ${ }^{20}$
Some authors suggested that one adenoma can induce the formation of another, mainly in cases of GH-secreting adenomas which release factors that may promote the proliferation of a secondary adenoma. ${ }^{1,7}$ Anyway, the role of pituitary and extrapituitary factors in inducing clonal expansion of genetically altered cells would be considered in the occurrence of multiple adenomas. In our patient, the existence of multicentric adenomas would be more plausible because the coexisting adenomas were completely separated from each other. In this context, the origin of the adenoma located within the stalk may be explained as a consequence of corticotroph basophilic invasion from the residual intermediate lobe into the posterior lobe. Starting from young adulthood, some corticotrophs in the zona intermedia proliferate into the posterior pituitary lobe and become more prominent with aging. A basophilic invasion has also been implicated as the possible origin of the extremely rare pituitary $\mathrm{ACTH}$ secreting adenomas arising from within the posterior lobe..$^{21,22}$

A poor surgical outcome has been reported in patients with double adenoma in whom the noncausative lesion was removed during the first operation. In the present case, hormonal data soon after surgery indicated the persistence of hypercortisolism and led the neurosurgeon to remove the lesion suspected at preoperative MR imaging of an enlarged infundibulum. The early occurrence of hypoadrenalism in the postoperative days indicated that a second corticotropinoma was resected: in fact, both tumors stained for ACTH on immunohistochemistry.

It is noteworthy in this regard that $\mathrm{ACTH}$-secreting adenomas originating in, or extending into, the pituitary stalk have been previously observed. ${ }^{23}$ These observations, together with the present case of double ACTH-producing adenomas, one in the anterior pituitary and the other attached to the pituitary stalk, once again emphasize that double ACTH-secreting adenomas may occur and that they need careful surgical management to recognize multiple lesions and to achieve a clinical resolution of Cushing's disease. On the other hand, the normalization of hormone hypersecretion cannot be achieved when the co-existing hypersecreting tumor is missed during surgery. In our case, the preoperative MR imaging of an enlarged infundibulum enabled us to discover, 
after the surgical failure, a second ACTH-secreting adenoma associated with a remnant of Rathke's pouch. To our knowledge, this is the first case of double ACTH-producing adenomas, one located in the anterior pituitary and the other attached to the pituitary stalk, reported in the literature.

\section{DISCLOSURE}

The authors have no financial conflict of interest.

\section{REFERENCES}

1. Kontogeorgos G, Scheithauer BW, Horvath E, et al, 1992 Double adenomas of the pituitary: a clinicopathological study of 11 tumors. Neurosurgery 31: 840-849.

2. Iacovazzo D, Bianchi A, Lugli F, et al, 2013 Double pituitary adenomas. Endocrine 43: 452-457.

3. Magri F, Villa C, Locatelli D, et al, 2010 Prevalence of double pituitary adenomas in a surgical series: clinical, histological and genetic features. J Endocrinol Invest 33: 325-331.

4. Kontogeorgos G, Kovacs K, Horvath E, Scheithauer BW, 1991 Multiple adenomas of the human pituitary. A retrospective autopsy study with clinical implication. J Neurosurg 74: 243-247.

5. Kim K, Yamada S, Usui M, Sano T, 2004 Preoperative identification of clearly separated double pituitary adenomas. Clin Endocrinol 61: 26-30.

6. Ratcliff JK, Oldfield EH, 2000 Multiple pituitary adenomas in Cushing's disease. J Neurosurg 93: 753-761

7. Sano T, Horiguchi H, Xu B, et al, 1999 Double pituitary adenomas: six surgical cases. Pituitary 1: 243-250.

8. Koutourousiou M, Kontogeorgos G, Wesseling P, Grotenhuis AJ, Seretis A, 2010 Collision sellar lesions: experience with eight cases and review of the literature. Pituitary 13: 8-17.

9. Syro LV, Horvath E, Kovacs K, 2000 Double adenoma of the pituitary: a somatotroph adenoma colliding with a gonadotroph adenoma. J Endocrinol Invest 23: 37-41.

10. Tomita T, Gates E, 1999 Pituitary adenomas and granular cells tumors. Incidence, cell type and location in 100 pituitary glands at autopsy. Am J Clin Pathol 111: 817825.

11. Tosaka M, Kohga H, Kobayashi S, et al, 2000 Double pituitary adenomas detected on preoperative magnetic resonance images. Case illustration. J Neurosurg 92: 361.

12. McKelvie PA, McNeill P, 2002 Double pituitary adenomas: a series of three patients. Pathology 34: 57-60.

13. Shimizu C, Koike T, Sawamura Y, 2004 Double pituitary adenomas with distinct histological features and immunophenotypes. J Neurol Neurosurg Psychiatry 75: 140.

14. Mohammed S, Cusimano MD, Scheithauer BW, Rotondo F, Horvath E, Kovacs K, 2010 O-Methylguanine-DNA methyltransferase immunoexpression in a double pituitary adenoma: case report. Neurosurgery 66: E421-E422.

15. Cannavò S, Curto L, Lania A, Saccomanno K, Salpietro FM, Trimarchi F, 1999 Unusual MRI finding of multiple pituitary adenomas in the gland: a case report and review of the literature. Magn Reson Imaging 17: 633-636.

16. Meij BP, Lopes MBS, Vance ML, Thorner MO, Laes ERJr, 2001 Double pituitary lesions in three patients with Cushing's disease. Pituitary 3: 159-168.

17. Pantelia E, Kontogeorgos G, Piaditis G, Rologis D, 1998 Triple adenoma in Cushing's disease: case report. Acta Neurochir 140: 190-193.

18. Oyama K, Yamada S, Hukuhara N, Hiramatsu R, Taguchi M, Imai Y, 2006 FSH-producing macroadenoma associated in a patient with Cushing's disease. Neuroendocrinol Lett 27: 733-736.

19. Andrioli M, Pecori Giraldi F, Losa M, Terreni M, Invitti C, Cavagnini F, 2010 Cushing's disease due to double pituitary ACTH-secreting adenomas: the first case report. Endocr J 57: 833-837.

20. Melmed S, 2003 Mechanism for pituitary tumorigenesis: the plastic pituitary. J Clin Invest 112: 1603-1618.

21. Hori A, Schmidt D, Kuebber S. 1999 Immunohistochemical survey of migration of human anterior pituitary cells in developmental, pathological, and clinical aspects: a review. Microscopy Research and Technique 46: $59-68$

22. Kuebber S, Ropte S, Hori A, 1990 Proliferation of adenohypophyseal cells into posterior lobe. Their normal anatomical condition and possible neoplastic potentiality. Acta Neurochir 104: 21-26.

23. Mason RB, Nieman LK, Doppman JL, Oldfield EH, 1997 Selective excision of adenomas originating in or extending into the pituitary stalk with preservation of pituitary function. J Neurosurg 87: 343-351. 